impetus for the development of epidemiological research (the study of the distribution and causes of disease in human populations) into the adverse health effects of the workplace.

Methods There are numerous epidemiological studies of specific industries, occupations and workplace 'exposures', from chemical, physical and biological agents to ergonomic factors and psychosocial stressors. These are usually observational in design and 'classical' intervention studies are much rarer. Occupational epidemiology plays an important role in identifying and quantifying risks and understanding the aetiology of disease and makes important contributions towards

i. risk and health impact assessment,

ii. setting standards/limits in workplaces and the general environment

iii. provision of evidence for compensation

iv. estimation of the burden of occupational disease to society.

Results Epidemiological studies of current risks from past exposure have directly informed strategic workplace risk reduction programmes and campaigns and production of guidance and practical interventions for stakeholders. Together with mechanistic information they contribute to occupational exposure limit (OEL) setting. More recent studies illustrate prediction modelling of the impact of reduction of OELs and $\mathrm{r}$ strategies such as improving compliance. Results from epidemiological studies are also incorporated into economic evaluations of risk options and this in turn has been important in decision making e.g. in the choice of EU OELs. International epidemiological studies can demonstrate important differences across nations in workplace exposures, resulting health consequences and use/lack of prevention measures.

Discussion Occupational epidemiology thus plays a vital role in increasing awareness of occupational disease and enumerating the impact of adverse working conditions and exposures. The occupational health community should continue to push for increased education on occupationally related ill-health, encourage routine collection of occupational data and, of course, persuade organisations to fund appropriate research.

\section{INFECTIONS IN THE WORKPLACE: IDENTIFYING PROBLEMS AND APPLYING RESEARCH TO PREVENTION}

Mary Ross. School of Public Health, University of the Witwatersrand, Johannesburg, South Africa

\subsection{6/oemed-2018-ICOHabstracts.31}

Introduction Infections are the only occupational diseases that can be transmitted from one worker to another. Although workplace and community-acquired infections have a long history of affecting health and productivity, from miners' 'consumption' and seafarers' plague to influenza and Ebola, occupational infections have been under-recognised, under-reported and under-researched. In most workplace settings, infectious diseases have not received the same attention as physical, chemical and psychosocial challenges. Similarly, workplaces have generally been underutilised in the prevention and control of infections. However, epidemics in the $21 \mathrm{st}$ century have evoked attention not only from the occupational health fraternity, but also from employers, workers and the media seeking policies and procedures to prevent and manage infection in the workplace.

Methods Selected infections and their impact in various occupational settings are explored to illustrate the challenges of their identification and management, as is the interface between public health and occupational health surveillance, research and interventions. The unique nature of infectious agents as an occupational hazard is considered, while longstanding and new public health research and strategies for prevention are evaluated in an occupational context.

Discussion A major challenge for research and prevention is measuring occurrence, morbidity or mortality from occupationally-acquired infections, especially when exposure in the workplace is not always recognised. Primary prevention interrupting the transmission cycle of micro- organisms comprises a variety of interventions that are implemented concurrently rather than in a hierarchy of control, while secondary prevention for affected individuals, becomes effective primary prevention for others. To date, even for health care workers, who dominate global research and interventions related to occupational infections, success has been somewhat limited to better resourced workplaces. It is vital for the focus to extend beyond the workplace in collaboration with public health care to promote research, recognition, prevention and management of infectious diseases for all workers.

\section{WORKING TOO MUCH, ANYWHERE AND AT ALL TIMES - WORKERS' HEALTH IN OUR CONTEMPORARY COMMUNICATION SOCIETY}

Lucia Rotenberg. Fiocruz, Oswaldo Cruz Institute, Laboratory of Health and Environmental Education, Rio de Janeiro, Brazil

\subsection{6/oemed-2018-ICOHabstracts.32}

The advancement of information and communication technologies (ICT) has changed spatial and temporal dimensions of work. Currently, in several occupations, there is a blurring of the boundaries between professional and private life, thus providing access to workers at any time of the day or night. This presentation will deal with consequences of ICT to workers' health and well-being, including the supplemental work from home, intensification of work, and the intrusion of work into individuals' personal life, affecting work life balance. Besides, the presentation will focus the phenomenon of constant connexion and its relation to a general feeling of time scarcity, also with implications to well-being. The view of organisations that care about working conditions, such as the Eurofound and the International Labour Office, will also be addressed, as well as policies to protect workers, such as the so-called 'right to be disconnected' recently implemented in France.

\section{OVERVIEW OF THE CURRENT STATE OF KNOWLEDGE ABOUT THE HEALTH EFFECTS OF NANOMATERIALS}

Paul A Schulte, Charles L Geraci, Eileen D Kuempel. National Institute for Occupational Safety and Health, Cincinnati, $\mathrm{OH}$, USA

\subsection{6/oemed-2018-ICOHabstracts.33}

Introduction It has been close to 20 years that engineered nanomaterials have entered commerce. There are tens of thousands of types of nanomaterials that have been produced and the hazard potential varies across them. Some may be hazardous and some not. The last decade of research has begun to identify important determinants of ENM toxicity but there is still much uncertainty about hazard potential. It is useful to 
attempt to sort out what currently is known and not about hazards of ENMs.

Methods The scientific literature from 2000-2017 was assessed using keywords and environmental scanning techniques to capture information about the health effects of ENMs. The focus will be on high volume ENM.

Result The extent of research health effects that have been identified for various high volume ENMs will be described. While many nanomaterials have been developed there are a limited number that are widely used in commerce. Common determinants of toxicity will be identified.

Discussion Assessing the hazard potential of ENM is a complex task since there are so many combinations of physiochemical parameters that may lead to ENMs having differential toxicity. The implications of this will be discussed as well the research needed to address the hazard potential of engineered of nanomaterials that are or could be in commerce.

\section{AIR PNEUMO: AN ACADEMIA-BASED QUALITY ASSURANCE OF PHYSICIANS' PROFICIENCY IN READING CHEST RADIOGRAPHS OF PNEUMOCONIOSIS}

Narufumi Suganuma. Department of Environmental Medicine, Kochi Medical School, Kochi University, Nankoku, Japan

\subsection{6/oemed-2018-ICOHabstracts.34}

Introduction Early detection of the disease, by routine standard chest X-ray, is one of the essential measures for secondary prevention. ILO has provided the guideline for radiographs reading for pneumoconioses according to the ILO classification to support the medical screening test and clinical purposes. The aim of this article is to describe the history and concept of the AIR Pneumo.

Methods Asian Intensive Reader of Pneumoconioses (AIR Pneumo) is a quality assurance program to train and certify physicians who works for prevention of pneumoconises, which is one of the major occupational health problems worldwide. The system is useful for epidemiological research, screening and surveillance of high-risk workers, diagnosis and compensation. Because of variability of reading by physicians, some organisation, e.g., US NIOSH, has developed the certification testing, called B -reader.

Result Since its first Bangkok workshop in 2006, AIR Pneumo has conducted 17 workshops 6 times in Thailand, 4 times in Brazil, twice in Japan, and one in each in Philippines and India. As Bangkok workshops invited international participants, most of physicians from ASEAN countries and D.R. Congo has attended the workshops.

Discussion The classification system holds its uniqueness in possessing standard radiographs that show profusion 0 to 3 for each types of small opacities, large opacities and pleural abnormalities. By using standard radiograph side-by-side to the subject radiographs that physicians are reading and classifying, higher inter reader agreement can be achieved. Understanding and using the classification properly demands certain amount of training. In accordance with WHO/ILO Global Programme for Elimination of Silicosis, ILO has been encouraging GPES participating nations to educate physicians to increase proficiency of reading radiographs of pneumoconioses. The AIR Pneumo, an academia based quality assurance of physicians proficiency in reading pneumoconiosis radiographs is sustainable approach with active involvement of local experts in GPES participant nations.
39 APPLYING HUMAN FACTORS TO PROMOTE A POSITIVE SAFETY CULTURE

Anna-Maria Teperi. Finnish Institute of Occupational Health, Helsinki, Finland

\subsection{6/oemed-2018-ICOHabstracts.35}

Introduction Safety management has traditionally been based on technical solutions and regulations. Although these traditional safety methods are still needed, they do not necessarily raise real safety levels. A prerequisite for improving safety is proper safety management, implemented by people who create and maintain safety through vigilance, competence, communication and group work, if the organisational structures around them support this human success. In this review, I summarise the research and development processes implemented in 2000-2017 to improve the safety, efficiency and well-being of organisations, from the aspect of human factors (HF) with safety critical domains.

Methods In 2000-2017, we conducted interviews, questionnaires and interventions among operative personnel, management and experts in the nuclear industry and the aviation, railway, and maritime sectors. Both business and authorities were represented. The HF tool was designed and modified for each field and the user experiences are presented here. The HF tool consists of a single tool, material for HF training, techniques for analysing cases at work, and proceedings for corrective actions. Its aim is to promote a positive safety culture.

Result The HF interventions helped the organisations handle incidents more transparently, giving way to more open discussions on demanding situations at work. The HF tool became more of a philosophy, with which to highlight and understand human performance at the individual, work, group and organisational level, also taking successes into account. It helped concreticise cognitive, work and organisational psychology as a facilitator of safety.

Discussion The HF tool promoted Safety-II thinking, which has recently been actively discussed as a safety paradigm shift, but has lacked the concrete tools to transform it from scientific debate to practice.

\section{6TH EUROPEAN WORKING CONDITIONS SURVEY: JOB QUALITY IN EUROPE}

Agnès Parent-Thirion, Isabella Biletta, Jorge Cabrita, Oscar Vargas, Greet Vermeylen, Alksandra Wiczynska, Mathijn Wilkens. Eurofound, Dublin, Ireland

\subsection{6/oemed-2018-ICOHabstracts.36}

Introduction The Union and Member states shall have as their objectives improved living and working conditions (article 151 of the TFEU). More and better job is an important policy objective for the European Union.

Methods Statistical analysis of the 6th European Working Conditions Survey. In 2015, the Sixth European Working Conditions Survey interviewed almost 44000 workers in 35 European Countries. Result Seven job quality indices are produced which at the level of the job, gathers these characteristics of work and employment that have been associated in positive or negative with health and wellbeing of workers. The job quality indices are: physical environment, social environment, work intensity, working time quality, skills and discretion, prospects and earnings. They are presented and discussed. Each index is associated with a positive experience of working life in health and well being, work life balance, engagement and motivation, financial security. The job quality indices are pooled together 